\title{
Estimation of the preventable proportion of congenital anomalies by selected risk factors in mothers: A case study in Iran
}

\author{
Saeed Dastgiri ${ }^{1}$, Leila R Kalankesh*2, ${ }^{* 2}$ egar Saberi ${ }^{3}$, Parisa Ziasarabi ${ }^{3}$ \\ Received: 30 Sep 2018 \\ Published: 15 Jul 2019
}

\begin{abstract}
Background: Worldwide, 1-6 percent of infants have at least one congenital anomaly that can lead to long-term consequences. This may have significant impacts on individuals, families, health-care systems, and societies. The aim of this study was to estimate the Population Attributable Fraction (PAF) of some congenital anomalies by three selected risk factors (obesity, diabetes and smoking) in the northwest of Iran and to estimate the number of preventable defects at birth in the population if we could reduce 50 percent of the exposure rate to these three risk factors at population level.

Methods: The regional data on smoking, obesity, and diabetes were entered in Population Estimate of Attributable Fraction of Congenital Conditions Everywhere (PEACE) software developed by International Clearinghouse for Birth Defects Surveillance and Research (ICBDSR). It calculates the PAF for congenital anomalies attributed to exposure to those three risk factors.

Results: This study showed that main anomalies attributed to "obesity" include Spina Bifida 40.1\% (95\% CI: 31.7-47.7), Hydrocephaly 26.8 (95\% CI: 9.3-42.3) and Anencephaly 17.4 (95\% CI: 1.6-31.9). The highest attributable defect to "smoking" was Cleft lip w/out Palate 5.8\% (95\% CI: 4.3-7.4), whereas for "diabetes" it was Tetralogy of Fallot 33.3 (95\% CI: 17.2-49.5). A similar pattern was found when assumed in the model, the impact of 50 percent reduction in the exposure to each of the risk factors on preventable cases of birth defects in the population.

Conclusion: Obesity, diabetes, and smoking in women of childbearing age increased the risk of occurrence of congenital anomalies. However, obesity and diabetes had a remarkably greater impact compared to smoking. More studies are needed to investigate the role of passive smoking as a risk factor for the occurrence of birth defects.
\end{abstract}

Keywords: Birth defects, Congenital anomalies, Obesity, Diabetes, Smoking

Conflicts of Interest: None declared

Funding: Iran Ministry of Health and Tabriz University of Medical Sciences

\section{*This work has been published under CC BY-NC-SA 1.0 license.}

Copyright $($ Iran University of Medical Sciences

Cite this article as: Dastgiri S, Kalankesh LR, Saberi N, Ziasarabi P. Estimation of the preventable proportion of congenital anomalies by selected risk factors in mothers: A case study in Iran. Med J Islam Repub Iran. 2019 (15 Jul);33:68. https://doi.org/10.47176/mjiri.33.68

\section{Introduction}

Congenital anomalies are defined as structural defects, chromosomal abnormalities, inborn errors of metabolism,

Corresponding author: Dr Leila R Kalankesh, Irkalankesh@tbzmed.ac.ir

1. Tabriz Health Services Management Research Centre, School of Medicine, Tabriz University of Medical Sciences, Iran

2. Tabriz Health Services Management Research Centre, School of Management and Medical Informatics, Tabriz University of Medical Sciences, Tabriz, Iran

3. School of Medicine, Tabriz University of Medical Sciences, Iran and hereditary disease diagnosed before, at, or after birth. Between 1 and 3 percent of newborns have a congenital

\section{$\uparrow$ What is "already known" in this topic:}

The etiology of the occurrence of congenital anomalies is still largely unknown. However it has been shown that selected groups of defects at birth may be prevented if exposure to three major common risk factors (including obesity, pre-gestational diabetes and smoking) are modified at population.

\section{$\rightarrow$ What this article adds:}

This study showed that main anomalies attributed to "obesity" include Spina Bifida 40.1\% (95\% CI: 31.7-47.7), Hydrocephaly 26.8 (95\% CI: 9.3-42.3) and Anencephaly 17.4 (95\% CI: 1.6-31.9). The highest attributable defect to "smoking" was Cleft lip w/out Palate 5.8\% (95\% CI: 4.3-7.4), whereas for "diabetes" it was Tetralogy of Fallot 33.3 (95\% CI: 17.2-49.5). 
disorder requiring medical attention $(1,2)$. These defects may result in long-term disabilities affecting children themselves, families, health system, and the community. Despite the considerable decrease in neonatal mortality in both developed and developing countries, congenital anomalies are still the leading cause of infant mortality (3, 4). According to some research reports, 17-42 percent of infant mortality has been attributed to congenital anomalies (1).

The etiology of the occurrence of congenital anomalies is still largely unknown (5-9). However, it has been shown that selected groups of defects at birth may be prevented if exposure to three major common risk factors (including obesity, pre-gestational diabetes, and smoking) are modified at the population (10-24).

The aim of this study was to estimate the Population Attributable Fraction (PAF) of some congenital anomalies by three selected risk factors (obesity, diabetes and smoking) in the northwest of Iran, and to estimate the number of preventable defects at birth in the population if we could reduce 50 percent of the exposure rate to these three risk factors at population level.

\section{Methods}

This study was carried out in the northwest region of Iran. Data for congenital anomalies were obtained from Tabriz Registry of Congenital Anomalies (TRoCA) for the birth year between 2005 and 2013. The total number of births, information on smoking, obesity, and pregestational diabetes were obtained for the same registry area from the provincial health system in the region .

Analysis of the data was performed using Population Estimate of Attributable Fraction of Congenital Conditions Everywhere (PEACE) software. This has developed and released by International Clearinghouse for Birth Defects Surveillance and Research (ICBDSR). PEACE calculates Population Attributable Fraction (PAF) of some common adverse pregnancy-related outcomes (i.e., the occurrence of congenital anomalies) for selected risk factors including obesity, diabetes, and smoking. PAF is defined as the proportion of the cases (i.e., birth defects) in the population that can be attributed to the specific exposures (i.e., obesity, diabetes, and smoking)(2).
In addition to PAF, we calculated the total frequency of defects at birth that could be avoided if we reduce 50 percent of exposure to those three risk factors in the community.

Confidentiality of all information on congenital anomalies was protected. Ethical clearance for the research was obtained from the ethics committee of Tabriz University of Medical Sciences.

\section{Results}

A total number of 160672 births for the years between 2005 and 2013 were recruited from the registry area of TRoCA from which 788 cases were diagnosed as having congenital anomalies.

Table 1 shows the prevalence of three major risk factors and the occurrence of selected congenital anomalies in the study population of the northwest region of Iran. In the area, obesity was the most prevalent risk factor (59.9 per 100) followed by smoking (18.1 per 100) and pregestational diabetes (9.1 per 100). The four most common congenital anomalies were Anencephaly (11.3 per 10000), Hydrocephaly (10.83 per 10000), Cleft lip w/out Palate (10.23 per 10000), and Anorectal Atresia (8.2 per 10000), respectively.

Table 2 presents the PAF, estimated number of cases of selected defects attributable to the exposure to three risk factors of obesity, smoking, and pre-gestational diabetes. This table also shows the estimated preventable cases of anomalies if it could be reduced by 50 percent of the exposure to these three risk factors at the population level.

Main anomalies attributed to "obesity" included Spina Bifida 40.1(95\% CI: 31.7-47.7), Hydrocephaly 26.8 (95\% CI: 9.3-42.3) and Anencephaly 17.4 (95\% CI: 1.6-31.9) whereas the highest attributable defect to "smoking" was Cleft lip w/out Palate 5.8\%; (95\% CI: 4.3-7.4), and for "diabetes" it was Tetralogy of Fallot 33.3 (95\% CI: 17.249.5). A similar pattern was found when assumed in the model the impact of 50 percent reduction in the exposure to each of the risk factors on preventable cases of birth defects in the population .Our finding indicated that if we reduce 50 percent of the exposure rate to three risk factors (obesity, diabetes, and smoking) at population level, we might be able to prevent a total of 96 cases of congenital

Table 1. Basic Information and Frequency of Selected Congenital Anomalies in Northwest of Iran

\begin{tabular}{lc}
\hline Congenital Anomalies & Rate (Per 10000) \\
\hline Anencephaly & 11.26 \\
Hydrocephaly & 10.83 \\
Cleft lip w/out Palate & 10.21 \\
Anorectal Atresia & 8.15 \\
Cleft Palate & 7.03 \\
Eye Defect & 4.42 \\
d-transposition of the Great Arteries & 4.05 \\
Spina Bifida & 1.56 \\
Hypoplastic Left Heart & 1.37 \\
Coarctation & 1.25 \\
Tetralogy of Fallot & 0.56 \\
Risk Factors & Rate (Per 100) \\
Obesity & 59.93 \\
Smoking & 18.13 \\
Pre-gestational diabetes & 9.07 \\
\hline Note: Data for congenital anomalies from Tabriz Registry of Registry Anomalies (TRoCA), and for obesity, \\
smoking and diabetes from provincial Health Centre
\end{tabular}




\begin{tabular}{|c|c|c|c|c|c|}
\hline & \multicolumn{2}{|c|}{$\begin{array}{l}\text { Population Attributable Fraction } \\
(95 \% \mathrm{CI})\end{array}$} & \multicolumn{2}{|c|}{$\begin{array}{l}\text { Estimated number of cases attribut- } \\
\text { able to the risk factor }(95 \% \mathrm{CI})\end{array}$} & \multirow[t]{2}{*}{$\begin{array}{l}\text { If reduced } 50 \% \text { estimated } \\
\text { cases prevented would be }\end{array}$} \\
\hline \multicolumn{5}{|l|}{ Obesity } & \\
\hline Anencephaly & 17.4 & $(1.6-31.9)$ & 31 & $(3-58)$ & 14 \\
\hline Spina Bifida & 40.1 & $(31.7-47.7)$ & 10 & $(8-12)$ & 4 \\
\hline Hydrocephaly & 26.8 & $(9.3-42.3)$ & 47 & $(16-74)$ & 20 \\
\hline Tetralogy of Fallot & 13.1 & $(4.6-21.2)$ & 1 & $(0-2)$ & 1 \\
\hline Hypoplastic Left Heart & 21.6 & $(11.0-31.7)$ & 5 & $(2-7)$ & 2 \\
\hline Coarctation of Aorta & 11.5 & $(0.5-21.6)$ & 2 & $(0-4)$ & 1 \\
\hline Cleft Platate & 10.6 & $(4.6-15.9)$ & 12 & $(5-18)$ & 6 \\
\hline Cleft lip w/out Palate & 6.6 & $(2.1-11.0)$ & 11 & $(3-18)$ & 5 \\
\hline \multicolumn{6}{|l|}{ Smoking } \\
\hline Eye Defect & 4.3 & $(2.0-6.8)$ & 3 & $(1-5)$ & 2 \\
\hline Cardiovacular Defect & 2 & $(0.4-3.7)$ & 2 & $(0-4)$ & 1 \\
\hline Cleft palate & 3.8 & $(1.8-6.0)$ & 4 & $(2-7)$ & 2 \\
\hline Cleft lip w/outPalate & 5.8 & $(4.3-7.4)$ & 9 & $(7-12)$ & 5 \\
\hline Anorectal Atresia & 3.5 & $(1.1-6.1)$ & 5 & $(2-9)$ & 3 \\
\hline \multicolumn{6}{|l|}{ Diabetes } \\
\hline d-transposition of the Great Arteries & 25.6 & $(13.3-39.8)$ & 17 & $(9-26)$ & 7 \\
\hline Tetralogy of Fallot & 33.3 & $(17.2-49.5)$ & 3 & $(2-4)$ & 1 \\
\hline Hypoplastic Left Heart & 19.7 & $(4.3-41.7)$ & 4 & $(1-9)$ & 2 \\
\hline Coarctation & 19.7 & $(6.0-36.7)$ & 4 & $(1-7)$ & 2 \\
\hline
\end{tabular}

anomalies related to obesity, 92 cases related to diabetes and 13 cases of birth defects related to smoking.

\section{Discussion}

In this study, we used the PEACE software to investigate the impact of three risk factors of obesity, pregestational diabetes, and smoking on the attributable rate of selected congenital anomalies in the registry area of Tabriz Registry of Congenital Anomalies in the northwest of Iran. Obesity was found to have the highest PAF mainly for Neural Tube Defects, diabetes for some congenital cardiac anomalies, and smoking mainly for Cleft defects. We showed that by reducing 50 percent of the exposure to three risk factors (obesity, diabetes, and smoking), a remarkable number of birth defects would be prevented in the population.

Watkins and colleagues studied the association between obesity and major congenital anomalies. They showed the higher risk of congenital anomalies incidence, particularly Neural Tube Defects, Spina Bifida in neonates of the obese women compared to offspring of those with normal weights (16). Our finding is also in accordance with the results reported in Stothard's study as they reported the significant association between obesity and incidence of congenital anomalies including Neural Tube Defects, Spina Bifida, Cardiovascular Defects, Cleft Palate, Cleft lip w/out Palate, Anorectal Atresia, and Limb Defects. However, according to their findings, the obese mothers had lower risks of neonates with Gastroschisis (17). Another cohort study has also reported the increased risk of birth defects among neonates of pregnant women with Body Mass Index (BMI) greater than 30 or less than 18 while no significant increased risk had been reported among those with BMI between 25 and 30. In particular, the findings have highlighted the increased risk of Interventricular Septal Defect, Cleft Lip, and Eye Defects among neonates of the obese women as well as the increased risk of Interatrial Septal Defects, Genital Anomalies, and Hypospadias among neonate of the mothers with low weights (18).
There is another report on the association (a range of weak to moderate) between the obesity and incidence of congenital structural anomalies including Spina Bifida, Congenital Heart Defects, Anorectal Atresia, Hypospadias, Limb Anomalies, Diaphragmatic Hernia, and Omphalocele. However, the likelihood of having an obese mother has been reported significantly low among the neonates with Gastroschisis (19).

The result of a systematic review reported no noticeable association between gestational diabetes and major congenital anomalies except a weak association for Congenital Heart Defects. However, one study has reported the significant association between the simultaneous existence of gestational diabetes and mother's pre-gestational BMI greater than 30, and Congenital Heart Defects and Neural Tube Defects. These conflicting results on the association between gestational pregnancy and major congenital anomalies might be attributed to the possible association of these defects with undiagnosed metabolic disorders (e.g. pre-gestational diabetes) related to the obesity (20). There are some evidence on a strong association between pre-gestational diabetes controlled by insulin and the risk of Kidney Agenesis, Obstructive Genitourinary Defects, Congenital Heart Defects, and Multiple Organ Defects. The association of pre-gestational diabetes with Neural Tube Defects, including Anencephaly and Encephalocele has also been reported in the literature $(16,21)$.

A significant association between mother's smoking habit and incidence of Cleft lip w/out Palate and Cleft Palate has been documented in the existing body of knowledge (22). Study of 173687 cases and 11.7 million controls showed a significant association between mother's smoking habit and occurrence of Cardiovascular Defects, Cleft Lip, Cleft Palate, and Eye Defects. However the impact of the smoking on Hypospadias was found to be protective in the study (13). Impact of indirect exposure of pregnant women to smoking on Cleft Lip with Palate and Cleft Palate has been reported in the findings of a recently published meta-analysis (23). Result of our study revealed that the highest attributable fraction of 
smoking was for Cleft lip w/out Palate, Eye Defects, and Cleft Palate. There was also an association between smoking and Anorectal Atresia as well as Heart Defects. These findings are similar to those reported in the studies of Little (24) and Hackshaw (13). There has also been a similar report on indirect smoking exposure of mothers and the association with congenital anomalies in the literature (23). However, they are not comparable with our findings as such mothers had not been included in our study.

Based on the results of our study, the highest attributable fraction of obesity was observed for Spina Bifida, Neural Tube Defects, and Hydrocephaly. These findings are in accordance with the results of a study conducted by Watkins as well as Stothard. There was also evidence of the association between high BMI and incidence of Eye Congenital Defects in the literature while this was not true for our findings. The association also was reported between the obesity of the pregnant women and incidence of major congenital defects such as Hypospadias, Limb Defect, Diaphragmatic Hernia, and Omphalocele while no such association was observed in our findings $(16,17)$.

According to the results of our study, the highest attributable fraction of diabetes was observed in Tetralogy of Fallot, d-transposition of Great Arteries, and Heart Defects. Our findings also showed the association between diabetes and Hypoplastic Left Heart Syndrome, Aorta Coarctation. These findings were not in line with the results of the study conducted by Parnell and colleagues in which they reported no association between diabetes and major congenital anomalies except for the Congenital Heart Diseases. However, results of the study by Nielsen have highlighted the strong association between pregestational diabetes and incidence of major congenital anomalies including Kidney Agenesis, and Obstructive Genitourinary Defects while such association was not found in our research $(20,21)$.

In this study, we examined risk factors of smoking, obesity, and diabetes in pregnant women without taking into account confounding factors such as mothers' age at pregnancy, their socio-economic status, current diseases, their medication and indirect exposures to smoking. This may be considered in further development of PEACE by ICBDSR.

\section{Conclusion}

Obesity, diabetes, and smoking in women of childbearing age increased the risk of occurrence of congenital anomalies. However, obesity and diabetes had remarkably greater impacts compared to smoking. More studies are needed to investigate the role of passive smoking and confounding factors on the occurrence of birth defects.

\section{Acknowledgments}

Authors wish to thank Iran Ministry of Health and Tabriz University of Medical Sciences for the financial support of this study.

\section{Conflict of Interests}

The authors declare that they have no competing interests.

\section{References}

1. Boyle B, Addor M, Arriola L, Barisic I, Bianchi F, Csaky-Szunyogh $\mathrm{M}$, et al. Estimating Global Burden of Disease due to congenital anomaly: an analysis of European data. Arch Dis Child Fetal Neonatal Ed. 2018;103:F22-F28

2. ICBDSR. PEACE tool. International Clearinghouse for Birth Defects Surveillance and Research 2018. Available at URL: http://www.icbdsr.org/resources/peace-tool/

3. Stone DH, Dastgiri S, Heidarzadeh M, Abdollahi HM, Imani S, et al. Uses, limitations, and validity of a registry of congenital anomalies in Iran: a critical review. J Environ Public Health. 2017; 2017.

4. Egbe A, Uppu S, Lee S, Ho D, Srivastava S. Congenital Malformations in the Newborn Population: A Population Study and Analysis of the Effect of Sex and Prematurity. Pediatr Neonatol. 2015; 56(1): 25-30

5. Christianson A, Howson C,Modell B. March of Dimes. Global report on birth defects: the hidden toll of dying and disabled children.New York: White Plains ; 2006.

6. Sass L, Urhoj S, Kjærgaard J, Dreier JW, Strandberg-Larsen K, NyboAndersen. Fever in pregnancy and the risk of congenital malformations: a cohort study. BMC Pregnancy Childbirth 2017;17(1): 413.

7. Pawluk M, Campaña H, Gili J, Comas B,Gimenez LG,Villalba MI, et al. Adverse social determinants and risk for congenital anomalies. Arch Argent Pediatr. 2014;112(3):215-223.

8. Dutta H, Baruah M,Borbora D. Maternal nutrition and the risk of congenital malformations in the tea garden community of Assam, Northeast India. CEGH. 2016; 4:63-68.

9. Kallen B. Genetic and non-genetic factors in the origin of congenital malformations, Epidemiology of human congenital malformations. 2014, Springer; pp 5-8.

10. EUROCAT: Primary Prevention of congenital anomalies. 2012.

11. Czeizel A. Birth defects are preventable. Int J Med Sci. 2005;2:9192.

12. Wahabi H, Fayed A, Esmaeil S, Mamdouh H, Kotb R. Prevalence and complications of pregestational and gestational diabetes in Saud Women: Analysis from Riyadh Mother and Baby Cohort Study (RAHMA). Biomed Res Int. 2017;2017.

13. Hackshaw A, Rodeck C,Boniface S. Maternal smoking in pregnancy and birth defects: a systematic review based on 173687 malformed cases and 11.7 million controls. Hum Reprod Update. 2011;17:589604.

14. Mills J. Malformations in infants of diabetic mothers. Birth Defects Res A Clin Mol Teratol 2010; 88:769-778

15. Chen C. Congenital malformations associated with maternal diabetes. Taiwan J Obstet Gynecol. 2005;44(1):1-7.

16. Watkins M, Rasmussen S, Honein MA, Botto LD, Moore, CA. Maternal Obesity and Risk for Birth Defects. Pediatrics. 2003;111(5):1152-1158.

17. Tothard K, Tennant P, Bell R, Rankin J. Maternal overweight and obesity and the risk of congenital anomalies: a systematic review and meta-analysis. JAMA. 2009;301:636-650.

18. Rankin J, Tennant P, Stothard K, Bythel M, Summerbell CD, Bell R. Maternal body mass index and congenital anomaly risk: a cohort study.

Int J Obes (Lond). 2010;34:1371-1380.

19. Waller D, Shaw G, Rasmussen S, Hobbs CA, Canfield MA, SiegaRiz AM, et al. Prepregnancy obesity as a risk factor for structural birth defects. Arch Pediatr Adolesc Med 2007;161:745-750.

20. Parnell A, Correa A,Reece E. Pre-pregnancy Obesity as a Modifier of Gestational Diabetes and Birth Defects Associations: A Systematic Review. Matern Child Health J. 201721:1105-1120.

21. Nielsen G, Norgard B, Puho E, Rothman KJ, Sorensen HT, Czeizel AE. Risk of specific congenital abnormalities in offspring of women with diabetes. Diabet Med. 2005;22:693-696.

22. Garne E, Loane M, Dolk H, Barisic I, Addor MC, Arriola L, et al. Spectrum of congenital anomalies in pregnancies with pregestational diabetes. Birth Defects Res A Clin Mol Teratol. 2012;94:134-140.

23. Sabbagh H, Hassan M, Innes N, Elkodary HM, Little J,Mossey PA. Passive smoking in the etiology of non-syndromic orofacial clefts: a systematic review and meta-analysis. PloS One. 2015;10:e0116963.

24. Little J, Cardy A,Munger R. Tobacco smoking and oral clefts: a meta-analysis. Bull World Health Organ. 2004; 82:213-218. 